COMMENT. The left medial temporal region is involved in memory and learning of novel stimuli and also during incremental learning and the consolidation or "binding' of new memories. These findings based on PET studies are supportive of the etiological importance of left temporal lobe arachnoid cysts associated with attention deficit and learning disorders, the TLAC/ADHD syndrome (Millichap JG. Neurology May 1997;48:1435).

\title{
BRAIN SIZE OR BRAIN ORGANIZATION AND INTELLIGENCE
}

The relationship of head size and intelligence quotient (IQ) to neuroanatomic similarities in young, healthy adult monozygotic (MZ) twins was examined using MRI and quantitative image analysis at the Department of Neurobiology, Harvard Medical School, Boston, MA. Significant effects of genotype but not birth order were found for the following measures: forebrain volume, cortical surface area, callosal area, head circumference, and full-scale IQ. Forebrain volume, cortical surface area, callosal area, and each brain measure and head circumference were correlated, but not IQ (Tramo MJ, Loftus WC, Stukel TA et al. Brain size, head size, and intelligence quotient in monozygotic twins. Neurology May 1998;50:1246-1254). (Reprints: Dr MJ Tramo, Dept of Neurobiology, Harvard Medical School, 220 Longwood Ave, Boston, MA 02115).

COMMENT. Brain measures in monozygotic twins are correlated with head circumference and brain volume but not with intelligence quotients. Intellectual similarities in MZ twins are not related to genetic influences on brain size ("bigger is 'not' better") but rather, brain organization. Intellectual functions are correlated with regional brain measures and local geometry of folds in the left cerebral cortex and forebrain, areas that govern language and abstract reasoning.

\section{FRONTAL LOBE DAMAGE AND MEMORY}

Skin conductance responses (SCRs) were recorded in a patient with frontal lobe damage and in normal control subjects while attempting recognition of famous and unfamiliar faces. The patient had more overt false recognition errors and misidentifications of unfamiliar faces than the controls, while, similar to controls, he showed accurate covert autonomic discrimination of familiar from unfamiliar faces. Overt and covert forms of memory for face recognition are dissociable following frontal lobe damage. Memory distortions are related to a sense of familiarity, or deja vu, that have a resemblance to previously seen faces. The false recognition results from breakdown of frontal memory retrieval mechanisms involved in correctly attributing familiar faces to the appropriate source. (Rapcsak SZ, Kaszniak AW, Reminger SL et al. Dissociation between verbal and autonomic measures of memory following frontal lobe damage. Neurology May 1998;50:1259-1265). (Reprints: Dr Steven Z Rapczak, Neurology Section (I-127), VA Medical Center, 3601 South 6th Ave, Tucson, AZ 85723).

COMMENT. False recognition and confabulation of memory and misattributions of familiarity with faces are characteristics of frontal lobe dysfunction. Overt or explicit and covert implicit measures of memory are dissociable. Verbal and autonomic measures of recognition are poorly correlated in patients with agnosia and amnesia.

Prosopagnosia or agnosia for faces may be found in lesions of the posterior portion of the minor hemisphere, and unlike those with frontal lobe damage, patients fail to recognize familiar individuals, insisting that all faces are unfamiliar. Prosopagnosia is associated with contralateral visual field defect. 